\title{
The Visual Interface of the STAR Framework ${ }^{1}$
}

\author{
Ronaldo S. Mello, Lia G. Golendziner, Flávio R. Wagner \\ Universidade Federal do Rio Grande do Sul, Instituto de Informática \\ Caixa Postal 15064, 91501-970 Porto Alegre RS, Brasil \\ e-mail \{mello,lia,flavio\}@inf.ufrgs.br
}

\begin{abstract}
Visual data management employed in EDA Frameworks helps in many design tasks, offering various facilities, specially related to the formulation of queries. In the STAR framework, a visual interface is the primary user interface to the system, allowing access to different managers and offering facilities for browsing and querying design objects. A graphical browser is available, allowing the navigation through design objects stored in the database, such that object properties can be shown and related objects can be investigated, departing from a selected one. Graphical queries can be formulated in an interactive way. Complex queries can be formulated via a textual SQL-like query language, extended with facilities for version manipulation and navigation in the inheritance hierarchy defined by the STAR data model.
\end{abstract}

\section{Introduction}

Data management in eletronic design automation (EDA) frameworks usually includes design object modelling, complex object manipulation, design alternatives representation and version control. Databases, in this kind of application, store objects with different types of relationships: composition, representation and versioning [1]. These relationships raise several query possibilities, useful in different stages of the design process.

From a database point of view, query formulation is usually done in a textual way. The disadvantage of a textual query language is that it does not apply to new or infrequent users considering that a good knowledge of the database schema and query language syntax is needed. This problem is being solved with the development of visual technology which provides user-friendly graphic interfaces and visual languages, allowing object visualization and manipulation in a graphical way.

Visual data management employed to EDA frameworks helps in many design tasks, offering various facilities, specially related to the formulation of queries. Database browsing, for example, allows the investigation of descendants in a representation hierarchy. Object selection can be provided by an interactive query formulation. In this case, the designer can describe query conditions by selecting object properties, conditional symbols and constant values, for example. All these facilities and other

\footnotetext{
${ }^{1}$ This work is partially supported by CNPq and CAPES.
} 
ones are available in the visual interface of the STAR framework, under development at Universidade Federal do Rio Grande do Sul.

This paper is organized as follows. Section 2 describes the features and the data model of the STAR framework. In section 3, the visual interface is presented and its browsing and query formulation facilities are exemplified. Section 4 is dedicated to the STAR textual query language, section 5 compares the visual interface with other EDA framework interfaces and, finally, conclusions and future works are discussed in section 6.

\section{The STAR Framework}

STAR is an open system that supports integration of several tools aimed at different tasks, architectures and design technologies [2]. The STAR data model allows the definition of object schemata in a hierarchical way, where schema nodes can be generalizations of other nodes.

The root node in this inheritance hierarchy is a Design object. Each Design gathers an arbitrary number of ViewGroups and Views. Each ViewGroup may in turn gather, according to defined criteria, any number of other ViewGroups and Views. Views are of one of three types: HDL Views, for behavioral descriptions, MHD Views, for structural descriptions, and Layout Views, for physical descriptions [2,3]

Associated to each View, there is a graph of ViewStates, where each ViewState contains the real design data, in one of the various design representations (layouts, HDL descriptions, and so on). Each ViewState inherits attributes from the corresponding View and its ascendants in the inheritance hierarchy (upwards to the Design node). A ViewState can be described as a composition of sub-objects, and each of these may reference objects that are represented by their own object schemata.

All nodes of the object schemata can be versioned, and the Version Manager automatically keeps track of the inheritance relationships among these versions. Design version management in STAR is described elsewhere [3].

Object schemata can evolve in time, and new schemata can be defined dynamically, departing from an existing schema and making changes to it [4]. Schema evolution is maintained through versions, so that version management is applied not only to the design objects themselves, but also to object schemata.

A configuration is defined for each Viewstate as a selection of a particular version of a particular design object for each sub-object within the ViewState. Besides static and dynamic configurations, the STAR framework also supports open configurations (as in VHDL). The Configuration Manager [5] is a special module that allows specification of configurations either in an interactive way or through an application programming interface.

\section{The Visual Interface}

The visual interface is the primary user interface of the STAR framework, allowing access to different managers and offering facilities for browsing and querying design objects in a graphical or textual way 
[6].

Figure 1 shows the visual interface architecture and its communication with the other STAR components. The visual query module is responsible for processing interface commands or events and returning objects and messages to be visualized. The designer interacts with the visual interface either through navigation (browsing actions) or formulating graphical queries. Both forms are converted to a textual query specification which is sent to the textual query module. In addition, textual queries can be directly formulated by designers which are familiar with its syntax. The textual query syntax is described in the section 4.

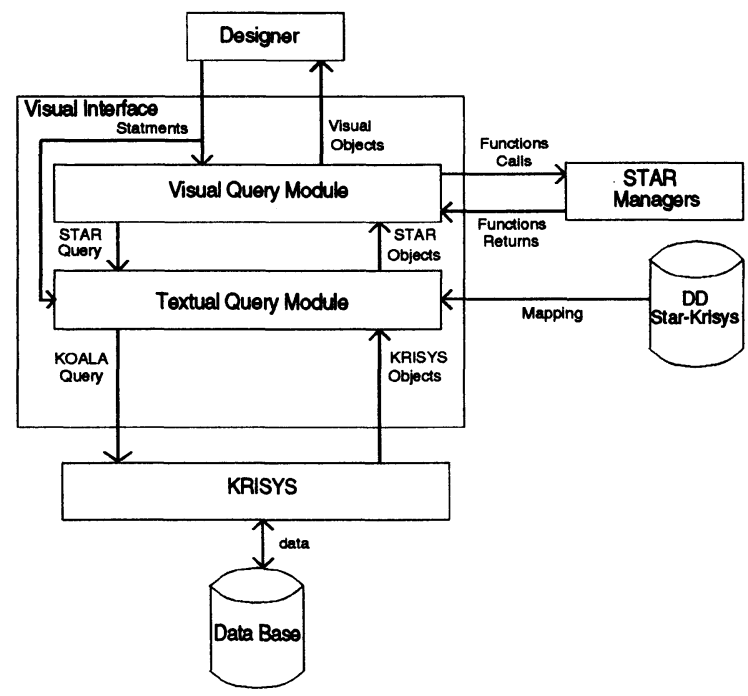

Figure 1: Visual interface architecture and its communication with the other STAR components.

The textual query module, in your turn, verifies textual queries syntax and translates it to the database query language (KOALA language). The STAR data management is supported by the system KRISYS, a knowledge base management system [7]. KRISYS processes a KOALA statment and returns a query result to the textual query module. A data dictionary STAR-KRISYS allows the mapping from STAR objects to KRISYS objects and vice versa. This data dictionary is accessed by the textual query module during the translation to KOALA statements as well as during the query result analysis. This analysis results in a query table (of STAR objects) which is sent to the visual query module to be displayed in the interface.

The visual query language is composed by a window hierarchy, representing the different levels of the object hierarchy in the STAR data model. Several windows can be opened allowing the visualization of many design object representations in the object schemata at the same time. All windows present a group of standard menu options and mouse events, providing a uniform interaction. Some interface operations are exemplified in the following subsections. 


\subsection{Browsing Facilities}

Browsing facilities include navigation through the object schemata, visualization of object properties, version selection, and query activation. Navigation through the object schemata is the most common operation performed by the designer, offering an interactive mechanism to traverse the object's definition following its several representations.

A short browsing session is described in the following to illustrate the interaction with the STAR framework through the visual interface.

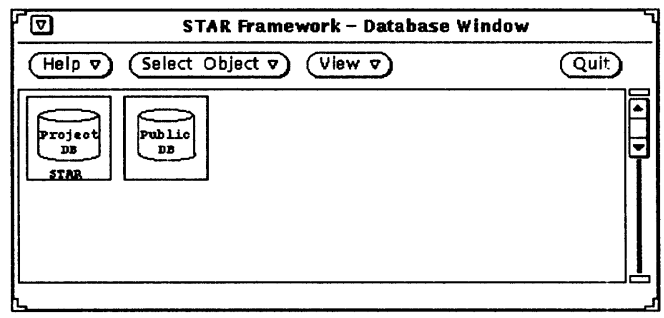

Figure 2: Database window.

The database window is opened when the visual interface is activated. This window displays Project databases accessible by the designer as well as the Public database (figure 2). The STAR project database can be selected (figure 3) leading to a project database window, where the designer's private database, together with two libraries objects called Risco and Calc, are shown.

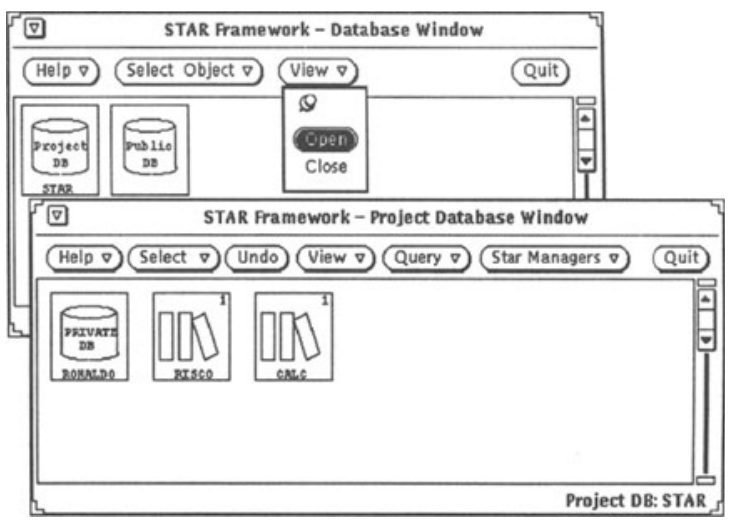

Figure 3: Project database window.

Different icons identify different object types. Library icons for example, are displayed as a group of books. When the designer knows the path to a specific object in the hierarchy, it is possible to visualize it by using the Select-Object menu option. Figure 4 shows the selection of the ViewGroup Slice (down to Design Op) related to the Library Risco, as described in the object line command. 


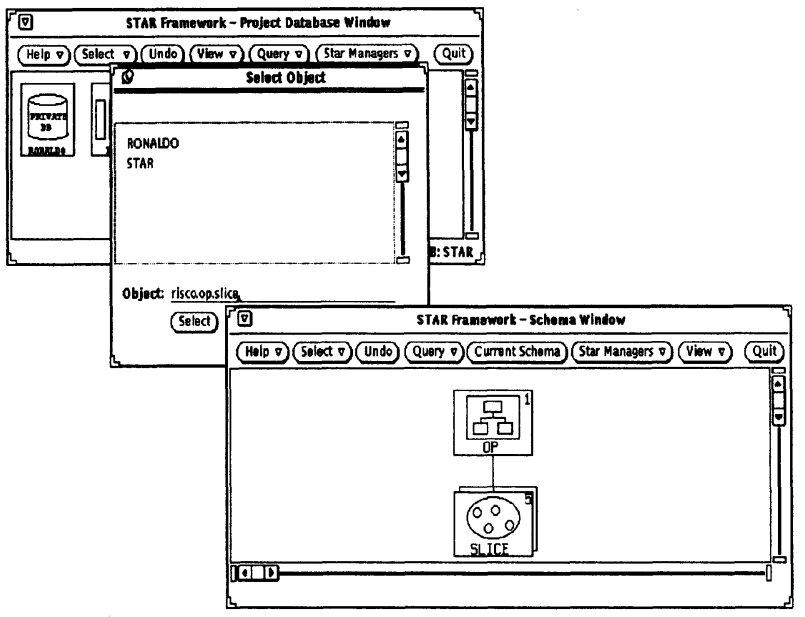

Figure 4: Schema window.

The schema window displays the objects selected. This window allows the visualization of the object schemata from the Design node down to View nodes.

Visual means are also employed to emphasize object versions. Next and previous versions are denoted by extra icons placed at the bottom-left and upper-right corners respectively (see Slice node in Figure 4). The number associated to each object icon indicates which node version may be currently investigated. Another version of the node can be investigated if its icon is selected with the mouse.

Visualization of object properties is provided by choosing a node with a specific mouse button. This action opens an object properties window. The properties of the Slice object are shown in Figure 5. Properties that are references to other objects are indicated by its value (the name of the referenced object) followed by an arrow. Once the arrow is selected, another object properties window is opened, showing the properties of the referenced object.

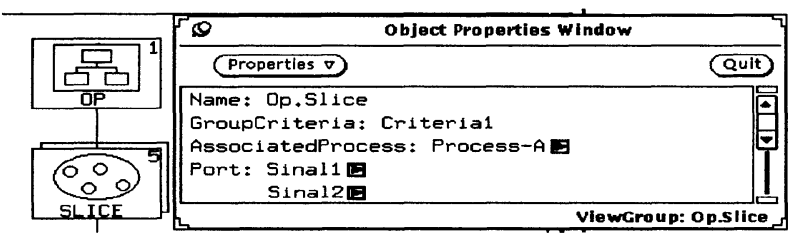

Figure 5: Object properties window.

Investigation of object descendants in the object schemata can be done by selecting an object icon. In figure 6 it is possible to see three Views (LO, ENL and Extracted) as the result of the selection on the node Slice.

In the same way, if LO node is chosen, a viewstate window is opened, showing the ViewState called VsLO1 and its two derived ones: VsLO2 and VsLO5 (figure 7). 


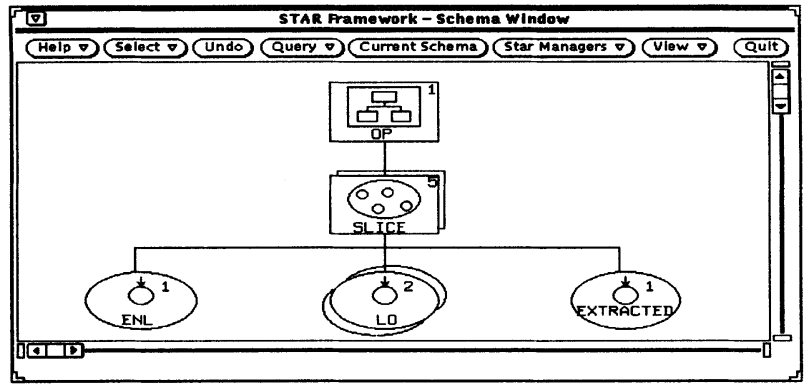

Figure 6: Visualization of Slice descendants.

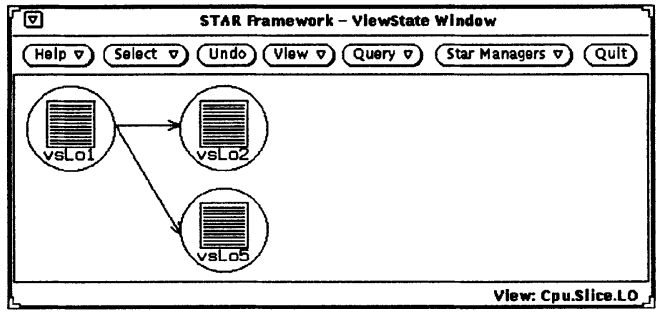

Figure 7: ViewStates graph related to View $\mathbf{L O}$.

\subsection{Query Facilities}

Queries can be formulated while browsing the database, for example, to restrict the selection of descendants or versions of a specific node. Queries can be defined in a graphical (interactive) or textual way. Through a query menu, which can be opened when a node is selected, the designer can choose the query formulation type.

Graphical queries allow the definition of simple predicates associated to a single object type. When the graphical option is selected from the query menu, the designer initially chooses the kind of relationship, like versions or descendants. In case of descendants, another menu question the designer about the descendant object type intended to be queried. After this, it is still possible to choose between retrieving all or some instances of the selected object type. If the second option is choosen, a graphical query window is opened to help the designer in the formulation of query conditions. An example of the sequence described above can be shown in figure 8, considering that the designer intends to search for some Views related to the ViewGroup Slice.

Query conditions are defined in an easy way in the graphical query window. Object type properties, conditional symbols and constant values can progressively be chosen to define query conditions that can be combined by logical operators. Figure 8 also exemplifies a condition, displayed at the Query line command, that retrieves Views which present Ports with direction in.

The Query line command can optionally be used by the designer to specify conditions in a textual way. Query conditions can be modified, either to update it or to remove it completely. 


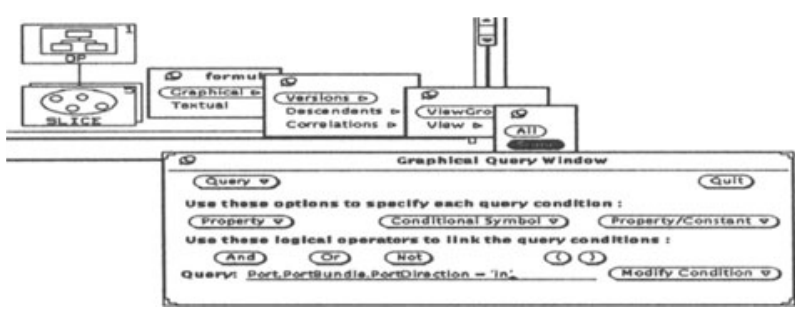

Figure 8: Specifying a graphical query.

Textual queries are defined using a textual query editor. In the next subsection the STAR textual query language is described through some examples.

Graphical and textual query answers can be shown either graphically or textually. In the first case, the objects retrieved by the query are displayed in an iconic form, together with their inheritance and versioning relationships to other objects. In the second case, a query table window is opened and the user can navigate on it.

It is possible to save query descriptions and query results after query formulation. Query descriptions can be further loaded in the graphical query window or textual query editor and re-used in new queries. Stored query results can be selected (menu option Query-SavedResults) and other queries can be formulated over them to retrieve some of its instances (incremental query facility).

\section{The textual query language}

Queries can also be formulated by using the textual query language of the STAR framework. The textual query language is an SQL-based language with extended constructs for version manipulation and predicates that deal with complex attributes and relationships in the inheritance hierarchy. Figure 9 shows the basic structure of the language.

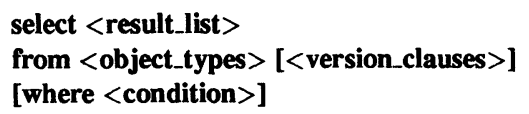

Figure 9: Textual query language structure.

Object versions can be queried by using specific clauses linked to an object variable in the from clause. These clauses are: firstversion, lastversion, predversion, succversion and versions. Except for the versions clause, that retrieves a set of versions, all other clauses return a single node version of the STAR object schemata. The query described in figure 10, for example, returns Op.Slice versions which holds the GroupCriteria named Criterial.

Some object attributes can be defined as references to a single object or a set of objects in the database, being called complex attributes. In the first case, it is possible to define conditions over the referenced object attributes by using a dot notation. Manipulation of set attributes is provided by the 


\author{
select $v_{0}^{*}$ \\ from viewgroup vg versions $v$ \\ where vg.Name = 'Op.Slice' \\ and v.GroupCriteria = 'Criteria1'
}

Figure 10: Using versions clause.

clauses in and contains, which tests element-set and set-set relationships, respectively. The following query (figure 11), looks for Port names of the View Op.Slice.ENL with direction out. Port is a set attribute of a View object type. In addition, these Views must be associated to Process called Process1. AssociatedProcess is a reference to a object type Process.

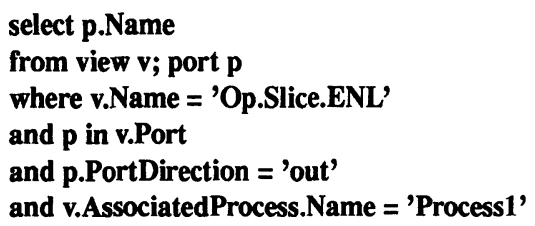

Figure 11: A query predicate holding complex attributes.

Queries dedicated for retrieving object descendants or the father of a specific node are formulated by using the clause is descendant of. A query example is shown in figure 12. It returns all ViewGroups names that are descendants of the Design Op. The optional character '*' means to search for all ViewGroups descendants in the subtree under Op.

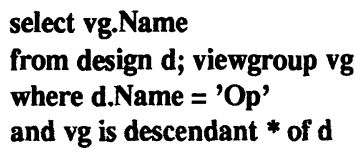

Figure 12: A query predicate that tests relationships in the inheritance hierarchy.

Saved query results can also be queried, providing incremental queries facility. Query results names are refered in the from clause preceded by the character '@'. Supposing a query that retrieves data about ViewStates was stored with the name Vs_result, a new query, described in figure 13, returns ViewStates names from Vs_result that present the ViewDescription file ga.

\title{
5 Comparison with other interfaces
}

Compared to other EDA framework interfaces, the visual interface of the STAR framework offers more facilities related to query formulation.

Nelsis design platform [8] provides meta data and design objects visualization and manipulation. Textual queries can be formulated interactively by selecting object types and attributes in the meta data interface and specific browsers makes possible navegation through different kinds of design object relationships. However, it does not allow graphical queries formulation in the same way of the STAR 


\author{
select vs.Name \\ from @Vs_result vs \\ where vs.ViewDescription = 'file_ga'
}

Figure 13: An incremental query.

visual interface, which is completely free from a textual syntax. Visualization of various design objects at the same time, provided by the STAR visual interface, is not an available facility either in Nelsis platform.

CADLAB desktop [9] offers a hierarchical browser to investigate a specific design object. Nodes can be selected and pre-defined methods may be applied to it. New windows can be opened for visualization of children nodes. The browser described by Gedye and Katz [10] allows graphical investigation of composition, versioning and equivalence relationships in a interconnected web of design objects. Nevertheless, query facilities are not supported by them, even in a textual way.

\title{
6 Conclusions and future works
}

This work describes browsing and query facilities supported by the visual query language of the STAR framework. A visual approach to the user interface helps the designer in understanding the complex structure of a design object, avoiding a deep knowledge of the STAR data model.

Most of the facilities available in database visual interfaces are taken into consideration by the STAR visual interface $[11,12,13,14]$ :

- schema visualization and manipulation (browsing);

- interactive (graphical) query formulation;

- textual query formulation for more specialized users;

- incremental queries;

- undo operation while browsing the database;

- facilities for new, as well as specialized users, in activation of operations;

- exhibition of different database objects at the same time (more than one window opened);

- uniformity of menu and mouse options in all windows of the interface;

- objects can be shown in different forms (icons, tables, and so on).

Currently, the visual interface is responsible for managing the browser and the formulation of queries. Communication with the STAR managers, for other design tasks related to data manipulation, is not still implemented.

Other future work concerns graphical queries. Complex queries formulation, which involves, for example, more than one object type or attribute projection in a query result, should be supported in order 
to increase graphical queries expression power. At present, complex queries can only be formulated in a textual way.

\section{Aknowledgements}

We greatfully aknowledge the work of Eduardo A. Rambo who made the implementation of the STAR visual interface windows, as well as the database objects visualization functions.

\section{References}

[1] T.J Barnes, D. Harrison, A.R. Newton, and R.L Spickelmier. Eletronic CAD frameworks. Kluwer Academic Publishers, 1992.

[2] F.R.Wagner, L.G.Golendziner, and M.R.Fornari. A tightly coupled approach to design and data management. In EURO-DAC. 1994.

[3] F.R.Wagner et al. Design version management in the STAR framework. In 3rd IFIP Intern. Workshop on EDA Frameworks. North-Holland, 1992.

[4] M.R.Fornari, L.G.Golendziner, and F.R.Wagner. Schema evolution in the STAR framework. Also to be presented at 4th IFIP Intern.Working Conf. on EDA Frameworks, 1994.

[5] F.R.Wagner. L.G.Golendziner, and H.G.Ribeiro. Configuration management in the STAR framework. Also to be presented at 4th IFIP Intern. Working Conf.on EDA Frameworks, 1994.

[6] R.S.Mello. A visual query languagefor the STAR framework. Porto Alegre, UFRGS, 1994. (Master's thesis, in Portuguese)

[7] N.M.Mattos. An approach to knowledge base management: requirements, knowledge representation and design issues. Universität Kaiserslautern, Fachbereich Informatik, 1989. (PhD thesis)

[8] P.Bingley and P.van der Wolf. A design platform for the NELSIS CAD framework. In 27th Design Automation Conference. ACM/IEEE, 1990.

[9] Cadlab. Cadlab Framework - Desktop 1.0 User's Guide. Paderborn, Cadlab, 1991.

[10] D. Gedye and R. Katz. Browsing the chip design database. In 25th Design Automation Conference. ACM/IEEE, 1988.

[11] H.Kim, H.F. Korth and A. Silberschatz. PICASSO: a graphical query language. Software Practice and Experience. v.13, n.3, 1990. p.169-203.

[12] C.T. Wu and D.K. Hsiao. Implementation of visual database interface using an object-oriented language. Visual Database Systems. Elsevier, 1989. p.105-125.

[13] M.Leong, S. Sam and D. Narasimhalu. Towards a visual language for an object-oriented multimedia database system. Visual Database Systems. Elsevier, 1989. p.465-495.

[14] R. Agrawal, N.H. Gehani and J. Srinivasan. Odeview: the graphical interface to Ode. In ACMSIGMOD International Conference on Managment of Data . ACM/IEEE, 1990. 